\title{
Retrospective analysis of the diagnostic effectiveness of the sentinel lymph node biopsy (SLNB) in vulvar
}

\author{
cancer \\ Szymon Piatek ${ }^{1} \mathbb{D}$, Wojciech Michalski ${ }^{2}$, Malgorzata Benke ${ }^{3}$, \\ Anna Danska-Bidzinska4 ${ }^{4}$, Mariusz Bidzinski ${ }^{1}$ (] \\ ${ }^{1}$ Department of Gynecologic Oncology, Maria Sklodowska-Curie National Research Institute of Oncology Warsaw, Poland \\ ${ }^{2}$ Department of Biostatistics, Maria Sklodowska-Curie National Research Institute of Oncology Warsaw, Poland \\ ${ }^{3}$ Department of Endocrinology and Nuclear Medicine, Maria Sklodowska-Curie National Research \\ Institute of Oncology Warsaw, Poland \\ ${ }^{4} 2^{\text {nd }}$ Clinic of Obstetrics and Gynecology, Medical University of Warsaw, Poland
}

\begin{abstract}
Objectives: Inguinal lymphadenectomy used in the treatment of vulvar cancer often results in complications, such as lymphoedema or abnormal wound healing. Aim of this study was assessment of the diagnostic effectiveness of the sentinel lymph node biopsy (SLNB) procedure in patients treated due to vulvar cancer.

Material and methods: Eighty-four patients diagnosed with squamous cell vulvar carcinoma (FIGO I-IV) underwent preoperative lymphoscintigraphy with technetium 99 to map sentinel lymph node. During surgery sentinel lymph nodes were identified and resected, followed by complete bilateral groin lymphadenectomy.

Results: Sentinel lymph nodes were mapped with lymphoscintigraphy and biopsied in $84.3 \%$ and $90.1 \%$ of patients, respectively. False negative predictive value (FNPV) was $9.1 \%$ and false negative rate (FNR) was recorded in $16.7 \%$ of cases. Patients in advanced stages (FIGO III and IV) had significantly lower rate of lymphatic mapping compared to those in stage I and II $(O R=0.148, p=0.022)$. Detection of sentinel lymph node in lymphoscintigraphy for tumor grade 2 and 3 was nearly eight times lower than for grade 1 cancers, however without statistical significance $(O R=0.126, p=0.058)$.

Conclusions: The use of SLNB should be limited to vulvar cancer patients in early clinical stages.

Key words: sentinel lymph node procedure; vulvar cancer; retrospective analysis
\end{abstract}

Ginekologia Polska 2020; 91, 7: 379-382

\section{INTRODUCTION}

Vulvar cancer accounts for about $5 \%$ of all female genital cancers [1]. In 2016, 490 new cases of vulvar cancer were diagnosed in Poland [2]. Lymph nodes involvement is considered as the most important prognostic factor in vulvar cancer [3].

Nowadays, sentinel lymph node biopsy (SLNB) is the standard procedure for surgical assessment of melanoma, breast cancer, penile cancer and female genital cancers, including vulvar cancer. Lymphoscintigraphy requires standardization for appropriate sentinel lymph nodes mapping. Intraoperative identification of sentinel node and histopathological examination are subsequent crucial steps in SLNB.
Implementing sentinel lymph node procedure in clinical practice requires systematic validation. The purpose of this study was a retrospective assessment of the diagnostic effectiveness of the SLNB in the first 7-year period after implementation this method into clinical practice in our department.

\section{MATERIAL AND METHODS}

The analysis included patients with vulvar cancer who were treated between 2001 and 2007. Patients were staged according to the FIGO 1994 classification. During this period 115 women were treated at the Department. 17 cases of stage III were treated with radio(chemo) therapy exclusively, 7 patients of stage IV underwent 
chemotherapy based on cisplatin and 5-fluorouracil, 3 patients were disqualified from treatment due to advanced disease and poor general condition. In 4 cases vulvar melanoma was diagnosed - all of these patients were not included in the analysis. Patients with histopathological diagnosis of vulvar squamous cell carcinoma only were eligible for the study. Finally, the analysis included a total of 84 patients.

The sentinel lymph node biopsy procedure was performed in all 84 patients. It consisted of preoperative lymphoscintigraphy, intraoperative detection and biopsy of a radioisotope identified sentinel node, and finally histopathological evaluation.

The SLNB procedure was performed according to the two-day (long) protocol: radioisotope was administered 16-20 hours before surgery. Preoperative lymphoscintigraphy with nano-colloid radiolabelled technetium isotope ${ }^{99 \mathrm{~m}} \mathrm{Tc}$ ) was used. The radioactive marker was administered subcutaneously in four injections around the tumor, at a dose of $74 \mathrm{MBq}(18.5 \mathrm{MBq} \times 4)$. Lymphoscintigraphy imaging was performed with planar technique after 2 hours, using a two-head gamma camera with a low-energy collimator, on the sensor $128 \times 128$. The energy window was adapted to the energy of the ${ }^{99 \mathrm{~m} T c}$ isotope radiation ( $140 \mathrm{keV}$ ) that was used in the procedure. The data obtained was developed using dedicated workstations. The result of the radioisotope examination was transmitted to the operating team. During the surgery, lymph node with the largest isotope uptake in the groins was identified with manual gamma camera. The lymph node was considered as sentinel if isotopic activity in the wound decreased at least three times after its removal. Then a bilateral inguinal lymphadenectomy was performed. In cases with the lack of sentinel node localization, a complete groin lymphadenectomy was performed. Lymph nodes considered as sentinel were sent separately for histopathological examination. This evaluation was carried out with immunohistochemical staining with cytokeratin 7 and 19 .

Sentinel lymph node was considered as involved if metastasis was found.

Non-sentinel lymph nodes were considered as to be involved if metastasis was found in at least 1 non-sentinel lymph node.

The medium percentage of lymph nodes detected during the surgery as well as sensitivity, specificity, positive and negative predictive values (PPV, NPV) were estimated. The estimates are given along with $95 \%$ confidence intervals. Factors that could possibly affect the detection of sentinel node were also examined. For this purpose, the logistic regression model was used. Grading (G), clinical stage, age, the largest dimension and localization of the tumor were included in this model. To optimize the analysis, continuous variables have been categorized.
Effectiveness of sentinel lymph node identification in lymphoscintigraphy was assessed and potential factors that could affect it were examined. Compatibility of lymphoscintigraphic and surgical sentinel nodes detection was estimated.

All hypotheses were tested at a level of statistical significance of 0.05 .

Standard descriptive statistics tools are used to describe the study material. The statistical analysis was carried out with the IBM SPSS Statistics 23 statistical package. Due to the retrospective character of the study, the consent of the bioethics committee was not required.

\section{RESULTS}

The study group consisted of 84 women. Mean age was 66 years old (18 to 94 , standard deviation: 14 ). The largest dimension of the tumor ranged from 5 to $90 \mathrm{~mm}$ (median: $35 \mathrm{~mm}$ ), interquartile range (IQR): 22-50. Characteristics of study group was presented in Table 1.

\section{Surgical and pathological evaluation of SLNB}

Sentinel lymph node was found in $90.1 \%$ of patients. The estimated sensitivity and specificity were 54.5\% (25.1$-83.9 \%)$, and $83.3 \%$ (73.9-92.7\%), respectively. The positive

\begin{tabular}{|c|c|c|c|}
\hline & & $\begin{array}{l}\text { Number } \\
\text { of patients }\end{array}$ & $\begin{array}{l}\text { Percentage } \\
\text { of patients }\end{array}$ \\
\hline \multirow{5}{*}{ FIGO (1994) } & IA & 1 & 1.2 \\
\hline & IB & 11 & 13.1 \\
\hline & II & 35 & 41.7 \\
\hline & III & 31 & 36.9 \\
\hline & IV & 6 & 7.1 \\
\hline \multirow{4}{*}{ Grading } & 1 & 29 & 36.7 \\
\hline & 2 & 37 & 48.1 \\
\hline & 3 & 12 & 15.2 \\
\hline & missing & 6 & \\
\hline \multirow{3}{*}{ Side } & right & 27 & 41.8 \\
\hline & left & 13 & 20.9 \\
\hline & midline & 44 & 37.3 \\
\hline \multirow{2}{*}{ Multifocal } & no & 79 & 94.1 \\
\hline & yes & 5 & 5.9 \\
\hline \multirow{3}{*}{ Age [years] } & $<62$ & 26 & 31.0 \\
\hline & $62-73$ & 30 & 35.7 \\
\hline & $>73$ & 28 & 33.3 \\
\hline \multirow{4}{*}{ Maximum tumor size [mm] } & $\leq 25$ & 31 & 37.3 \\
\hline & $25-45$ & 22 & 26.5 \\
\hline & $\geq 45$ & 30 & 36.1 \\
\hline & missing & 1 & \\
\hline
\end{tabular}


Table 2. The sensitivity ${ }^{1}$, specificity ${ }^{2}, \mathrm{PPV}^{3}, \mathrm{NPV}^{4}, \mathrm{FNPV}^{5}, \mathrm{FNR}^{6}$ of SLNB in 71 patients. All 84 patients underwent SLNB but in 13 women pathological results were imprecise. Therefore, these patients were not included in the analysis. In these cases, the histopathological report: (1) did not specify which lymph nodes were sentinel; (2) contained the total number of resected and metastatic lymph nodes without taking into consideration site of lymphadenectomy

\begin{tabular}{|c|c|c|c|c|c|}
\hline & & & Non-sent & nph node & \\
\hline & & & Negative & Positive & \\
\hline & & $\mathrm{N}$ & 50 & 5 & 55 \\
\hline & Negative & $\%$ within SLN & $90.9 \%^{4}$ & $9.1 \%^{5}$ & $100 \%$ \\
\hline & & $\%$ within non-SLN & $83.3 \%^{2}$ & $45.5 \%$ & $77.5 \%$ \\
\hline & & $\mathrm{N}$ & 10 & 6 & 16 \\
\hline Sentinel lymph nodes & Positive & $\%$ within SLN & $62.5 \%$ & $37.5 \%^{3}$ & $100 \%$ \\
\hline & & $\%$ within non-SLN & $16.7 \%^{6}$ & $54.5 \%^{1}$ & $22.5 \%$ \\
\hline & & $\mathrm{N}$ & 60 & 11 & 71 \\
\hline & Total & $\%$ within SLN & $84.5 \%$ & $15.5 \%$ & $100 \%$ \\
\hline & & $\%$ within non-SLN & $100 \%$ & $100 \%$ & $100 \%$ \\
\hline
\end{tabular}

Table 3. The parameters of the final logistic model for chance of lymph nodes detection in scintigraphy

\begin{tabular}{|l|l|l|l|l|l|l|}
\hline & Beta coefficient & SE of Beta & $\mathbf{p}$ & OR & \multicolumn{2}{|c|}{ 95\%-confidence interval } \\
\hline Stage G $>1$ & -2.071 & 1.093 & 0.058 & 0.126 & 0.015 & 1.075 \\
\hline Clinical stage (1994) $>2$ & -1.911 & 0.832 & 0.022 & 0.148 & 0.029 & 0.755 \\
\hline Constant & 4.527 & 1.230 & $<0.001$ & 92.2 & & \\
\hline
\end{tabular}

and negative predictive values (PPV and NPV) were $37.5 \%$ (13.8-61.2\%) and 90.9\% (83.3-98.5\%), respectively. False negative predictive value (FNPV) was recorded in $9.1 \%$ of patients and false negative rate (FNR) was found in $16.7 \%$ of patients. Detailed statistical analysis was presented in Table 2. All analysed factors (i.e. age, maximum tumor size, staging, grading) were not significant for intraoperative sentinel lymph node detection $(p>0.1)$.

\section{Lymphoscintigraphy assessment}

Lymphoscintigraphy revealed sentinel lymph node in $84.3 \%$ (76.5-92.1\%) of patients. Patients with FIGO stage I and II were almost 7 times more likely to find sentinel lymph

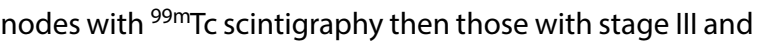
IV $(O R=0.148, p=0.022)$. Correlation between histological grading and lymphoscintigraphy was close to the statistical significance level $(p=0.058)$. Chance for sentinel lymph node detection in tumors $\mathrm{G} 2$ and $\mathrm{G} 3$ compared to $\mathrm{G} 1$ was almost eight times lower $(\mathrm{OR}=0.126)$. Logistic model analysis was presented in Table 3.

\section{Comparison of lymphoscintigraphy and surgery}

There was no correlation between lymphoscintigraphy outcome and surgical evaluation of sentinel lymph node site. The estimated values of kappa coefficient for the left and right side were $0.192(p=0.0638)$ and $0.048(p=0.628)$, respectively.

\section{DISCUSSION}

The status of regional lymph nodes is the most essential of all known risk factors for vulvar cancer recurrence [4]. The assessment of lymphatic drainage in vulvar cancer is important, especially in fact that in clinical stage I and II metastases to regional nodes occur at $10.7 \%, 26.2 \%$, respectively [5]. In the past, surgical treatment of the vulvar cancer relied on en bloc resection of the vulva and inguinal lymph nodes. Postoperative period after such disabling surgeries was complicated by long wound healing, infections and psychological problems. In the 1970s, surgical management of vulvar cancer resection was modified to triple incision technic. It has improved postoperative period significantly, especially wound healing and reducing long hospitalization stay.

In mid 1990s, SLNB was introduced in vulvar cancer [6, 7]. Lymphoscintigraphy with ${ }^{99 \mathrm{~m} T c}$ was the most regularly used technique with high accuracy of mapping lymphatic drainage [8-10]. Therefore since 2000, we used this method in our Department to perform SLNB in patients with vulvar cancer.

The study analysed data covering the period between 2001 and 2007; the eligibility criteria for SLNB in vulvar cancer patients were not established. At that time in our department, all patients with malignant vulvar neoplasms were qualified to SLNB, regardless of tumor size (in 32 patients tumor size was $>4 \mathrm{~cm}$, and the largest tumor dimension was $90 \mathrm{~mm}$ ), abnormal inguinal lymph in physical examination and imaging. $90.1 \%$ of patients had at least one sentinel lymph node found 
during operation. This result may be disappointing regarding study of Vidal-Sicart et al. [11], who found sentinel lymph nodes in $98 \%$ of patients. However, our results are acceptable regarding that SLNB has just been introduced as a new technic in those days and wide eligibility criteria for the procedure. Learning curve in SLNB as well as developing lymphoscintigraphy technique certainly had an impact on such result [12].

None of analysed factors (grading, staging, age, the maximum dimension and localization of the tumor) were relevant in intraoperative sentinel lymph node detection. However, in the meta-analysis, Hassanzade et al. [13] showed that efficacy of sentinel lymph node identification depended on the maximum tumor size and was $7 \%$ higher in tumors less than $4 \mathrm{~cm}$. The total efficacy of sentinel lymph node detection was $94.4 \%$ (regarding to patient) and $84.6 \%$ (regarding to a groin) [13].

Currently, the most regularly used criteria (ESGO and NCCN recommendations) for sentinel lymph nodes detecting include: 1) tumors less than $4 \mathrm{~cm}, 2$ ) clinically not enlarged groin lymph nodes and 3) lack of abnormal imaging $[14,15]$. Nowadays, according to these criteria, only selected patients are qualified to SLNB in our department. The recommendations are the results of the clinical trial - GROINSS V [16]. Patients suffering from squamous vulvar cancer with a maximum tumor diameter $<4 \mathrm{~cm}$ were qualified to this study. False negative predictive value was $2.9 \%$ in the trial. In our analysis, FNPV was 3 times higher and reached $9.1 \%$. Probably, this was due to the qualification of patients regardless of tumor size $-36.1 \%$ of patients had tumors $>4.5 \mathrm{~cm}$ in diameter. It may be caused by complete blockage of lymphatic vessels by cancer cells in large tumors [13]. Results of the GOG 173 study confirmed this thesis - FNPV for tumors $4-6 \mathrm{~cm}$ was $7.4 \%$, while in tumors $<4 \mathrm{~cm}$ decreased to $2 \%$ [17]. In this prospective study FNR was $8.3 \%$, compared to $16.7 \%$ presented in our paper. That is an argument for qualifying patients with tumors up to $4 \mathrm{~cm}$ for the procedure of sentinel node detection. According to Levenback et al. [17] FNR can be reduced by using radiocolloid and dye.

We found that staging $(p=0.022)$ and tumor grading $(p=0.058)$ may be related to efficacy of the lymphoscintigraphy. In the literature, no analysis has been found regarding clinicopathological factors and the lymphoscintigraphy results.

The study was a retrospective analysis and had some limitations. Staging of vulvar cancer was conducted according to FIGO classification from 1994. Another problem was related to definition of SLNB. A sentinel lymph node identified by gamma probe have radioactivity at least ten times higher than the background counts. This definition comes from practice guideline in gynecological cancers of The European Association of Nuclear Medicine published in 2014 [18]. In our study, covering the years 2001-2007, radioactivity at least three times the background was adopted to mark the node as SLN. This could affect the results.

\section{CONCLUSIONS}

These results became starting point for improving SLNB in clinical practice and conducting further research in this area. In our opinion, SLNB should be limited to patients with vulvar cancer in early clinical stages.

\section{REFERENCES}

1. Hauspy J, Beiner M, Harley l, et al. Sentinel lymph node in vulvar cancer. Cancer. 2007; 110(5): 1015-1023, doi: 10.1002/cncr.22874, indexed in Pubmed: 17626265 .

2. Wojciechowska U, Czaderny K, Ciuba A. et al. Cancer in Poland in 2016. Warsaw 2018.

3. Woelber L, Mahner S, Voelker K, et al. Clinicopathological prognostic factors and patterns of recurrence in vulvar cancer. Anticancer Res. 2009; 29(2): 545-552, indexed in Pubmed: 19331201.

4. Frey JN, Hampl M, Mueller MD, et al. Should Groin Recurrence Still Be Considered as a Palliative Situation in Vulvar Cancer Patients?: A Brief Report. Int J Gynecol Cancer. 2016; 26(3): 575-579, doi: 10.1097/IGC.0000000000000637, indexed in Pubmed: 26894938.

5. Berek J, Hacker N. Practical Gynecologic Oncology, 3rd ed. Lippincott Williams \& Wilkins, Baltimore 2000

6. Ansink AC, Sie-Go DM, van der Velden J, et al. Identification of sentinel lymph nodes in vulvar carcinoma patients with the aid of a patent blue V injection: a multicenter study. Cancer. 1999; 86(4): 652-656, doi: 10.1002/(sici)1097-0142(19990815)86:4<652::aid-cncr14>3.0.co;2-r, indexed in Pubmed: 10440693.

7. de Hullu JA, Hollema H, Piers DA, et al. Sentinel lymph node procedure is highly accurate in squamous cell carcinoma of the vulva. J Clin Oncol. 2000; 18(15): 2811-2816, doi: 10.1200/JCO.2000.18.15.2811, indexed in Pubmed: 10920128.

8. Merisio C, Berretta R, Gualdi M, et al. Radioguided sentinel lymph node detection in vulvar cancer. Int J Gynecol Cancer. 2005; 15(3): 493-497, doi: 10.1111/j.1525-1438.2005.15314.x, indexed in Pubmed: 15882175.

9. Sideri M, De Cicco C, Maggioni A, et al. Detection of sentinel nodes by lymphoscintigraphy and gamma probe guided surgery in vulvar neoplasia. Tumori. 2000; 86(4):359-363, indexed in Pubmed: 11016730.

10. Rob L, Robova $H$, Pluta $M$, et al. Further data on sentinel lymph node mapping in vulvar cancer by blue dye and radiocolloid Tc99. Int J Gynecol Cancer. 2007; 17(1): 147-153, doi: 10.1111/j.1525-1438.2007.00806.x, indexed in Pubmed: 17291246.

11. Vidal-Sicart S, Puig-Tintoré LM, Lejárcegui JA, et al. Validation and application of the sentinel lymph node concept in malignant vulvar tumours. Eur J Nucl Med Mol Imaging. 2007; 34(3): 384-391, doi: 10.1007/s00259-006-0237-9, indexed in Pubmed: 17006693.

12. McMasters KM, Wong SL, Chao C, et al. University of Louisville Breast Cancer Study Group. Defining the optimal surgeon experience for breast cancer sentinel lymph node biopsy: a model for implementation of new surgical techniques. Ann Surg. 2001; 234(3): 292-9; discussion 299, doi: 10.1097/00000658-200109000-00003, indexed in Pubmed: 11524582.

13. Hassanzade M, Attaran M, Treglia G, et al. Lymphatic mapping and sentinel node biopsy in squamous cell carcinoma of the vulva: systematic review and meta-analysis of the literature. Gynecol Oncol. 2013; 130(1): 237-245, doi: 10.1016/j.ygyno.2013.04.023, indexed in Pubmed: 23612317.

14. Oonk MHM, Planchamp F, Baldwin P, et al. European Society of Gynaecological Oncology Guidelines for the Management of Patients With Vulvar Cancer. Int J Gynecol Cancer. 2017; 27(4): 832-837, doi: 10.1097/IGC.0000000000000975, indexed in Pubmed: 28441255.

15. NCCN Clinical Practice Guidelines in Oncology. Vulvar cancer version 2.2019. https://www2.tri-kobe.org/nccn/guideline/gynecological/english/vulvar.pdf (20.01.2020).

16. Van der Zee AGJ, Oonk MH, De Hullu JA, et al. Sentinel node dissection is safe in the treatment of early-stage vulvar cancer. JClin Oncol. 2008; 26(6): 884-889, doi: 10.1200/JCO.2007.14.0566, indexed in Pubmed: 18281661.

17. Levenback CF, Ali S, Coleman RL, et al. Lymphatic mapping and sentinel lymph node biopsy in women with squamous cell carcinoma of the vulva: a gynecologic oncology group study. J Clin Oncol. 2012; 30(31): 37863791, doi: 10.1200/JCO.2011.41.2528, indexed in Pubmed: 22753905.

18. Giammarile F, Bozkurt MF, Cibula D, et al. The EANM clinical and technical guidelines for lymphoscintigraphy and sentinel node localization in gynaecological cancers. Eur J Nucl Med Mol Imaging. 2014; 41(7): 14631477, doi: 10.1007/s00259-014-2732-8, indexed in Pubmed: 24609929. 